\title{
A. G. D. Watson's principal directions for a Riemannian $V_{t}$
}

\author{
By .H. S. Ruse.
}

(Received 12th April, 1945. Read 4th May, 1945.)

A. G. D. Watson (1939-41), remarking that there are no Ricci principal directions at a world-point of space-time at which the Einstein equations $R_{i j}=\frac{1}{4} g_{i j} R$ are satisfied, shows how to define at any world-point a set of principal directions intrinsically related to the Riemann tensor $R_{i j k l}$ itself. These directions are unique except when the epace-time has any kind of rotational symmetry about the world-point.

Principal directions for any $V_{4}$ have also been defined in terms of the Riemann tensor by Kretschmann and Struik (see Struik, 1927-28). A related discussion is that of Churchill (1932), who confines himself, however, to 4-dimensional Riemannian spaces of positive-definite $d s^{2}$. $I$ have myself recently given a detailed account of the matter (Ruse, 1947) for a $V_{4}$ satisfying the Einstein condition but having any signature.

Watson's approach to the subject is so different from that of the previous authors that it is not easy to see whether there is any connection between his work and theirs. It is the purpose of this paper to analyse his theory, but for a $d s^{2}$ of any signature and not merely for one having the signature \pm 2 as in Relativity.

$\$ 1$ contains a summary of results, taken from my own paper (1947), which will be needed in the discussion of Watson's theory. In $\S 2$ it is shown that his tensor $\Pi_{i j k l}$ is simply expressible in terms of the conformal curvature tensor, and in $\S 3$ his principal directions are defined for a $V_{4}$ of any signature. The connection between his and the Kretschmann-Struik directions is obtained in $\S 4$.

\section{§1. Principal directions in an Einstein $V_{4}$.}

Let then the signature of $d s^{2}$ be unspecified, and for the present suppose $V_{4}$ to be such that

$$
\boldsymbol{R}_{i j}=\frac{1}{4} g_{i j} R(i, j=1, \ldots, 4) .
$$


At any point $\left(x^{i}\right)$ of $V_{4}$ take an arbitrary orthogonal ennuple $h_{a}^{i}$ such that

$$
g_{i j} h_{a}^{\mathbf{i}} h_{b}^{j}=g_{a b} \equiv \delta_{a b}
$$

$a, b, c, d$ being used for ennuplet and $i, j, k, \ldots$ for tensor suffixes. If $d s^{2}$ is not positive definite at $\left(x^{i}\right)$, some of the $h_{a}^{i}$ will be imaginary.

At $\left(x^{i}\right)$ form the ennuplet components of all tensors under consideration. Then by (1.2), the ennuplet fundamental tensor $g_{a b}$ is equal to the Kronecker delta. Consider the quadratic form

$$
\frac{1}{4} R_{a b c d} p^{a b} p^{c d} \text {, }
$$

where $p^{a b}$ is a simple bivector. Equated to zero, this gives the equation of the Riemann line-complex in the projective 3-space associated with $V_{4}$ at the point $\left(x^{i}\right)$. When $d s^{2}$ is indefinite, some of the components of $R_{a b c d}$ are purely imaginary unless they happen to be zero.

Replace the skew suffix-pairs $a b, c d$ by single Greek suffixes according to the scheme

$$
\left.\begin{array}{rrrrrr}
a b=23 & 31 & 12 & 14 & 24 & 34 \\
a= & 2 & 3 & 4 & 5 & 6
\end{array}\right\} .
$$

Greek suffixes will all run from 1 to 6 . Then the quadratic form (1.3) becomes $R_{a \beta} p^{\alpha} p^{\beta}$, where, for an Einstein space, the symmetric $6 \times 6$ matrix $R_{\alpha \beta}$ has the form ${ }^{1}$

$$
R^{a \beta}=\left[\begin{array}{cc}
P & S \\
S & P
\end{array}\right]
$$

$P$ and $S$ being symmetric $3 \times 3$ matrices, not necessarily real. Also, if

$$
g_{a b c d} \equiv g_{a c} g_{b d}-g_{a d} g_{b c}
$$

then the quadratic form

$$
\frac{1}{4} g_{a b c d} p^{a b} p^{c d}
$$

becomes $g_{\alpha \beta} p^{\alpha} p^{\beta}$, where

$$
g_{\alpha \beta}=g_{\beta \alpha}=\left[\begin{array}{cc}
I & O \\
O & I
\end{array}\right]
$$

$I$ and $O$ being respectively the unit and null $3 \times 3$ matrices. Further, if $\epsilon_{i j k l}$ is the dualising tensor of components $\pm \sqrt{ } g ; 0$ (imaginary when

I In a non-Einstein space $R_{\alpha \beta}$ is represented by a general symmetric $6 \times 6$ matrix and not by one of the particular form (1.5). 
the signature of $d s^{2}$ is $\pm 2, g$ then being negative), the quadratic form

becomes $\epsilon_{\alpha \beta} p^{a} p^{\beta}$, where

$$
\frac{1}{4} \epsilon_{a b c d} p^{a b} p^{c d}
$$

Now let

$$
\epsilon^{\alpha \beta}=\epsilon^{\beta a}=\left[\begin{array}{ll}
O & I \\
I & O
\end{array}\right]
$$

$\left.\begin{array}{lll}\chi^{1}=\frac{1}{\sqrt{ }-2}\left(p^{1}+p^{4}\right), & \chi^{2}=\frac{1}{\sqrt{ } 2}\left(p^{2}+p^{5}\right), & \chi^{3}=\frac{1}{\sqrt{ } 2}\left(p^{3}+p^{6}\right) \\ \chi^{4}=\frac{1}{\sqrt{ } 2}\left(p^{1}-p^{4}\right), & \chi^{5}=\frac{1}{\sqrt{2}}\left(p^{2}-p^{5}\right), & \chi^{6}=-\frac{1}{\sqrt{2}}\left(p^{3}-p^{6}\right)\end{array}\right\}$,

or, as one equation,

$$
\chi^{a}=K_{\beta}^{a} p^{\beta}
$$

where the matrix $K \equiv\left[K_{\beta}^{a}\right]$ is given by

$$
K=\frac{1}{\sqrt{ } 2}\left[\begin{array}{rr}
I & I \\
I & -I
\end{array}\right]
$$

(Ruse, 1947, §2). Obviously

$$
K=K^{t}=K^{-1},
$$

$t$ denoting the transpose. In terms of the $\chi$-variables, the quadratic form (1.3) becomes ( ${ }) R_{a \beta} \chi^{a} \chi^{\beta}$, where

$$
\begin{aligned}
{ }_{(x)} R_{\alpha \beta} & =R_{\gamma^{\delta}} \frac{\partial p^{\gamma}}{\partial \chi^{a}} \frac{\partial p^{\delta}}{\partial \chi^{\beta}} \\
& =R_{\gamma \delta} K_{\alpha}^{\gamma} K_{\beta}^{\delta}, \\
& =K^{t} R K
\end{aligned}
$$

in matrix notation, whence, by (1.5), (1.12) and (1.13),

$$
{ }_{(\mathrm{x})} R_{\mathrm{a \beta}}=\left[\begin{array}{cc}
P+S & O \\
O & P-S
\end{array}\right] \text {. }
$$

Similarly, for the quadratic forms (1.7) and (1.9),

$$
{ }_{(x)} g_{a \beta}=\left[\begin{array}{ll}
\dot{I} & O \\
O & I
\end{array}\right], \quad{ }_{(x)} \epsilon_{a \beta}=\left[\begin{array}{rr}
I & O \\
O & -I
\end{array}\right],
$$

$g_{a \beta}$ remaining invariant under the transformation and so retaining the form (1.8). It should be noted that the variables $\chi^{a}$ depend upon the initial choice of ennuple $h_{a}^{i}$. They will be described as belonging to $h_{a}^{i}$.

If the elementary divisors of the matrices $P \pm S$ are simple ${ }^{1}$, it

-1 This proviso is necessary because, although $P \pm S$ are symmetric, they are not necessarily real. 
is possible to find linear orthogonal transformations

$$
\left.\begin{array}{l}
\left(\chi^{1}, \chi^{2}, \chi^{3}\right) \rightarrow\left({ }^{\prime} \chi^{1}, \chi^{2},{ }^{\prime} \chi^{3}\right) \\
\left(\chi^{4}, \chi^{5}, \chi^{6}\right) \rightarrow\left({ }^{\prime} \chi^{4}, \chi^{5}, \chi^{6}\right)
\end{array}\right\}
$$

which respectively reduce $P+S, P-S$ to diagonal form. Because of the orthogonality of the transformations, the quadratic forms $\sum_{1,2,3}\left(\chi^{a}\right)^{2}$ and $\sum_{4,6}\left(\chi^{a}\right)^{2}$ are invariant, and the $g$ - and $\epsilon$-matrices therefore retain the forms (1.16). But the $R$-matrix becomes

$$
{ }_{\left({ }^{\prime} \mathrm{x}\right)} R_{a \beta}=\left[\begin{array}{cc}
M_{123} & O \\
O & M_{456}
\end{array}\right]
$$

where $M_{123}$ and $M_{456}$ are $3 \times 3$ diagonal matrices. Thus the $R-, g$ and $\epsilon$-matrices are all in diagonal form in the ' $\chi$-system. Their simultaneous reducibility to diagonal form is a characteristic property of an Einstein space.

To the transformation (1.17) corresponds in $V_{4}$ a rotation of the orthogonal ennuple $h_{a}^{i}$ to a new ennuple ' $h_{a}^{i}$, the ' $\chi^{a}$ being the variables that belong to ' $h_{a}^{i}$ in the sense defined above. This ennuple gives four directions in $V_{4}$ which are one set of Kretschmann-Struik principal directions for the Einstein space.

When the latent roots of the matrix $P+S$ are unequal, and those of $P-' S$ are also unequal, there are exactly six such sets of orthogonal directions. ${ }^{1}$ A change from one set to another in $V_{4}$ corresponds to a signed permutation ${ }^{2}$ of the variables $\left({ }^{\prime} \chi^{1},{ }^{\prime} \chi^{2},{ }^{\prime} \chi^{3}\right)$ or of $\left({ }^{\prime} \chi^{4},{ }^{\prime} \chi^{5},{ }^{\prime} \chi^{6}\right)$ among themselves.

When $d s^{2}$ is positive definite at $\left(x^{i}\right)$, all six of the orthogonal ennuples ' $h a$ define real directions of $V_{4}$. When the signature of $d s^{2}$ at $\left(x^{i}\right)$ is \pm 2 , exactly one of them gives real directions; and when the signature is zero, some or none may define real directions (Ruse, loc. cit., §4).

If the latent roots of $P \pm S$ are not all different, but their elementary divisors are simple, there are an infinite number of sets of orthogonal directions defined in this way. In that case the $V_{4}$ has some kind of symmetry about $\left(x^{i}\right)$. If the elementary divisors are not simple, there are no principal directions in the sense defined above.

1 There are also nine other sets of Kretschmann-Struik principal directions (Ruse, loc. cit., § 8). But in an Einstein space all nine consist of null directions, and we shall not be concerned with them in the present paper.

${ }^{2}$ A signed permutation is a transformation like ${ }^{4} \chi^{4}=-" \chi^{4},{ }^{\prime} \chi^{5}=-" \chi^{6},{ }^{\prime} \chi^{6}=-" \chi^{5}$, the signs being chosen so as to make it unimodular. 


\section{$\S 2$. Watson's tensor $\Pi_{i j k l}$.}

Now suppose $V_{4}$ not necessarily an Einstein space. Let

$$
\begin{aligned}
& \Delta_{i j k l}^{+} \equiv \frac{1}{4}\left(g_{i j k l}+\epsilon_{i j k l}\right), \\
& \Delta_{i j k l} \equiv \frac{1}{4}\left(g_{i j k l}-\epsilon_{i j k l}\right),
\end{aligned}
$$

where

$$
g_{i j k l} \equiv g_{i k} g_{j l}-g_{i l} g_{j k} \text {. }
$$

Watson's theory involves only one tensor $\Delta_{i j k l}$ explicitly, but two implicitly, the one being the complex conjugate of the other. It must be remembered that $\epsilon_{i j k l}$, as defined in this paper, is purely imaginary when the signature of $d s^{2}$ is \pm 2 , and $\Delta^{+}, \Delta^{-}$are then complex conjugates. A similiar remark applies to the tensors $\Pi^{+}, \Pi^{-}$below. They replace Watson's single tensor $\Pi$.

Let

$$
\begin{aligned}
& \Pi_{i j k l}^{+} \equiv \Delta_{i j m n}^{+} \Delta_{k l r s}^{+} R^{m n r s}+\frac{1}{6} \Delta_{i j k l}^{+} R, \\
& \Pi_{i j k l}^{-} \equiv \Delta_{i j m n}^{-} \Delta_{k l r s} R^{m n r s}+\frac{1}{6} \Delta_{\overline{i j k l}}^{-} R .
\end{aligned}
$$

Like $R_{i j k l}$, both $\Pi$-tensors are skew in $i, j$ and in $k, l$, are symmetric for an interchange of the pairs $i j, k l$, and satisfy the cyclic identity

$$
\Pi_{i j k l}+\Pi_{i k l j}+\Pi_{i l j k} \equiv 0 \text {. }
$$

Also, as is easy to prove, the Ricci tensors formed from them are both zero, that is, both satisfy

$$
\Pi_{i j} \equiv g^{m n} \Pi_{i m n j}=\mathbf{0} .
$$

Let now ${ }^{\circ} R_{i j k l}$ be the dual of $R_{i j k l}$ and $R_{i j(k l)}$ the "semi-dual" defined by

$$
\begin{aligned}
& { }^{\circ} R_{i j k l} \equiv \frac{1}{4} \epsilon_{i j m n} \epsilon_{k l p q} R^{m n p q}, \\
& R_{i j(k l)} \equiv R_{(k l) i j} \equiv \frac{1}{2} \epsilon_{k l p q} R_{i j} \cdot p q,
\end{aligned}
$$

the suffixes on the right being raised in the usual way by means of $g^{i j}$. Then a simple calculation shows that

$$
\Pi_{i j k l}^{+}=\frac{1}{4}\left(R_{i j k l}+{ }^{\circ} R_{i j k l}+R_{i j(k l)}+R_{(i j) k l}\right)+\frac{1}{\frac{1}{4}}\left(g_{i j k l}+\epsilon_{i j k l}\right) R \text {. }
$$

Now (Ruse, 1944, p. 71)

$$
{ }^{\circ} \dot{R}_{i j k l}=R_{i j k l}+\left(g_{i k} R_{j l}+g_{j l} R_{i k}-g_{i l} R_{j k}-g_{j k} R_{i l}\right)-\frac{1}{2} g_{i j k l} R,
$$

and, if $C_{i j k l}$ is the conformal curvature tensor (Eisenhart, 1926, p. 90), then

$$
C_{i j k l}=R_{i j k l}+\frac{1}{2}\left(g_{i k} R_{j l}+g_{j l} R_{i k}-g_{i l} R_{j k}-g_{j k} R_{i l}\right)-\frac{1}{6} g_{i j k l} R .
$$

The last two equations give

$$
C_{i j k l}=\frac{1}{2}\left(R_{i j k l}+{ }^{\circ} R_{i j k l}+\frac{1}{6} g_{i j k l} R\right),
$$


A. G. D. Watson's principal directions for a Riemannian $V_{4} 149$ whence

$$
\begin{aligned}
C_{(i j) k l} & \equiv \frac{1}{2} \epsilon_{i j m n} R_{. . \dot{k} l}^{m n} . \\
& =\frac{1}{2}\left(R_{(i j ; k l}+R_{i j(k l)}+{ }_{i j}^{1} \epsilon_{i j k l} R\right) .
\end{aligned}
$$

From (2.9), (2.12) and (2.13) it follows that

Similarly

$$
\Pi_{i j k l}^{+}=\frac{1}{2}\left(C_{i j k l}+C_{(i j) k l}\right) \text {. }
$$

$$
\Pi_{i j k l}=\frac{1}{2}\left(C_{i j k l}-C_{(i j) k l}\right) \text {. }
$$

The apparent asymmetry of these in the pairs of suffixes $i j, k l$ is accounted for by the fact that

as is obvious from (2.13).

$$
C_{(i j) k l}=C_{i j(k l)} \text {, }
$$

\section{§3. Watson's principal direclions.}

The tensor $C_{i j k l}$ has the same symmetry properties for interchanges of its suffixes as the Riemann tensor, and also satisfies the cyclic identity. Moreover, the Ricci tensor formed from it is zero, that is,

$$
C_{i j} \equiv g^{m n} C_{i m n j}=0 .
$$

It therefore satisfies the Einstein condition (with zero " cosmological constant") even though the Riemann tensor itself may not do so. So, if we start as in $\S 1$ with an arbitrary orthogonal ennuple $h_{a}^{i}$, forming the ennuplet ${ }^{1}$ components $C_{a b c l}$ of $C_{i j k l}$ and then replacing suffix-pairs by single Greek suffixes, we obtain for $C_{a \beta}$ a matrix of the form (1.5), say

$$
C_{a \beta}=\left[\begin{array}{ll}
A & B \\
B & A
\end{array}\right],
$$

where $A, B$ are symmetric $3 \times 3$ matrices. From $C_{(i j) k l}$ we therefore obtain

$$
C_{(a) \beta} \equiv g_{a \gamma} \epsilon^{\gamma \delta} C_{\delta \beta} .
$$

But the matrix $\epsilon^{\gamma \delta}$ is equal to its reciprocal $\epsilon_{a \beta}$, so by (1.8) and (1.10),

$$
\begin{aligned}
C_{(a) \beta} & =\left[\begin{array}{ll}
I & O \\
O & I
\end{array}\right] .\left[\begin{array}{ll}
O & I \\
I & O
\end{array}\right]\left[\begin{array}{ll}
A & B \\
B & A
\end{array}\right] \\
& =\left[\begin{array}{ll}
B & A \\
A & B
\end{array}\right] .
\end{aligned}
$$

I 'This introduction of an ennuplet system corresponds to Watson's use of locally galilean coordinates. 
Hence by (2.14), (2.15), (3.2) and (3.4),

$$
\begin{aligned}
& \Pi_{\alpha \beta}^{+}=\frac{1}{2}\left[\begin{array}{cc}
A+B & A+B \\
A+B & A+B
\end{array}\right], \\
& \Pi_{\alpha \beta}^{-}=\frac{1}{2}\left[\begin{array}{cr}
A-B & -(A-B) \\
-(A-B) & A-B
\end{array}\right]
\end{aligned}
$$

If we now transform to the $\chi$-variables of (1.11) by pre-multiplying and post-multiplying by the matrix $K$ [cf. (1.14) and (1.15), with reference to (1.13)], we obtain

$$
\begin{aligned}
& { }_{(x)} \Pi_{a \beta}^{+}=\left[\begin{array}{cc}
A+B & O \\
O & O
\end{array}\right], \\
& { }_{(x)} \Pi_{a \beta}^{-}=\left[\begin{array}{cc}
O & O \\
O & A-B
\end{array}\right] .
\end{aligned}
$$

Thus

$$
{ }_{(x)} \Pi_{a \beta}^{+} x^{\alpha} \chi^{\beta}, \quad{ }_{(x)} \Pi_{\alpha \beta}^{-} \chi^{\alpha} \chi^{\beta}
$$

are ternary quadratic forms in $\left(\chi^{1}, \chi^{2}, \chi^{3}\right)$ and $\left(\chi^{4}, \chi^{5}, \chi^{6}\right)$ respectively. If these are simultaneously reduced to sums of squares by orthogonal transformations $\chi \rightarrow{ }^{\prime} \chi$ of the type (1.17), which can be done when the elementary divisors of the matrices $A \pm B$ are simple, we obtain, in $V_{4}$, a rotation of the ennuple $h_{a}^{i}$ to a new ennuple ' $h_{a}^{i}$ in the manner described in $\S 1$ for an Einstein space. Having found one such, signed permutations of $\left({ }^{\prime} \chi^{3},{ }^{\prime} \chi^{2},{ }^{\prime} \chi^{3}\right)$ or of $\left({ }^{\prime} \chi^{4},{ }^{\prime} \chi^{5},{ }^{\prime} \chi^{6}\right)$ lead to five other essentially distinct ennuples of the same type. The six sets are unique when the latent roots of $A \pm B$ are unequal; otherwise $\left(x^{i}\right)$ is a point about which $V_{4}$ has some kind of symmetry, and the number. of ennuples is infinite. To avoid the need for constant reference to the latter possibility, it will hereafter be assumed that the latent roots are all different.

The six ennuples give six sets of Watson principal directions. It will be seen below that only one is real in the case considered by him, namely when $d s^{2}$ has signature \pm 2 .

The principal directions as thus dofined are non-existent when the conformal curvature tensor is zero. If it is zero everywhere in $V_{4}$, then $V_{4}$ is conformal to a flat space (Eisenhart, 1926, p. 92). They are also non-existent when the elementary divisors of $A . \pm B$ are not simple.

It may be noted that Watson's tensor $P_{i j k l}$, defined to be twice the real part of his tensor $\Pi_{i j k l}$, is minus the conformal tensor $C_{i j k l}$, and that he takes the Riemann tensor with sign different from that of the present paper. 
A. G. D. Watson's principad directions for a Riemannian $V_{4} 151$

§4. Identification of Watson's directions.

Still supposing that $V_{4}$ is not necessarily an Einstein space, let

$$
G_{i j k l .} \equiv \frac{1}{2}\left(R_{i j k l}+{ }^{\circ} R_{i j k l}\right) \text {. }
$$

This, so to speak, is the self-dual part of the Riemann tensor, since obviously

$$
{ }^{\circ} G_{i j k l}=G_{i j k l} .
$$

The last equation is equivalent to

$$
G_{i j}=\frac{1}{4} g_{i j} G,
$$

where $G_{i j} \equiv g^{m n} G_{i m n j}, G \equiv g^{i j} G_{i j}=R$ (Ruse, 1944, p. 71).

By (2.12),

$$
G_{i j k l}=C_{i j k l}-\frac{1}{1^{2}} g_{i j k l} R,
$$

and so, forming ennuplet components with respect to an arbitrary ennuple $h_{a}^{i}$ and proceeding as before, we have, by (3.2) and (1.8),

$$
G_{\alpha \beta}=\left[\begin{array}{ll}
A^{\prime} & B \\
B & A^{\prime}
\end{array}\right],
$$

where

$$
A^{\prime}=A-\frac{1}{12} R I \text {. }
$$

Pre- and post-multiplying by the matrix $K$ of (1.12), we obtain

$$
{ }_{(\alpha} G_{\alpha \beta}=\left[\begin{array}{cc}
A^{\prime}+B & O \\
O & A^{\prime}-B
\end{array}\right] .
$$

This is the equation for the tensor $G_{i j k l}$ corresponding to equation (1.15) for the Riemann tensor in the case when the latter satisfies the Einstein condition.

Now the orthogonal transformations $\chi \rightarrow^{\prime} \chi$ that reduce ${ }_{(\chi)} G_{a \beta}$ to diagonal form are exactly the same as those of $\S 3$ which reduce $\Pi_{a \beta}^{+}$ and $\Pi_{a \beta}^{-}$to diagonal form. For by (4.6),

$$
A^{\prime} \pm B=A \pm B-\frac{1}{12} R I
$$

and the matrix $I$ is invariant under an orthogonal transformation. Hence Watson's principal directions are the non-null KretschmannStruik directions for the self-dual part $G_{i j k l}$ of the Riemunn tensor.

When the Riemann tensor itself satisfies the Einstein condition, it is self-dual, and so

whence

$$
\begin{aligned}
{ }^{\circ} R_{i j k l} & =R_{i j k l}, \\
G_{i j k l} & =R_{i j k i} . \\
R_{i j} & =\frac{1}{4} g_{i j} R,
\end{aligned}
$$

Therefore when 
Watson's directions are the non-null Kretschmann-Struik directions themselves.

Whether $R_{i j k l}$ is self-dual or not, it follows from the work quoted in $\S 1$ that there are six sets of these directions, real or imaginary (according to the signature of $d s^{2}$ ) as there described.

\section{REFERENCES.}

Churchill, R. V., 1932. "On the geometry of the Riemann tensor," Trans. Amer. Math. Soc., vol. 34, pp. 126-152.

Eisenhart, L. P., 1926. Riemannian geometry, Princeton.

Ruse, H. S., 1944. " On the line-geometry of the Riemann tensor," Proc. Roy. Soc., Edinburgh, vol. 62, pp. 64-73.

_-, 1947 (?). “The self-polar Riemann complex for a $V_{4}$," Proc. London Math. Soc. In press.

Struik, D. J., 1927-8. " "On sets of principal directions in a Riemannian manifold of four dimensions," Journ. Math. Phys., Mass. Inst. Tech., vol. 7, pp. 193-197.

Watson, A. G. D., 1939-41. "Principal directions in a gravitational field," Proc. Edinburgh Math. Soc. (2), vol. 6, pp. 12-16.

\section{UnIVERsity College,} SoUthamptoN. 